\section{rev Psi}

Revista de Psicología (UNLP)

https://revistas.unlp.edu.ar/revpsi

\title{
El malentendido como oportunidad. Una cuestión de palabras
}

\author{
Lucila Romero ${ }^{1,2}$ \\ Julieta Gentile ${ }^{1}$ \\ Marina Vega'1,3 \\ Correspondencia \\ lucilaromero@gmail.com

\section{Filiaciones institucionales} \\ ${ }^{1}$ Colegio de Psicologos/as de la Provincia de $\quad{ }^{2}$ HIGA San Martín, La Plata (Argentina) \\ Buenos Aires - Distrito XI (Argentina) \\ ${ }^{3}$ Subsecretaría de Derechos Humanos de la Pcia. de \\ Buenos Aires (Argentina)
}

\section{Resumen}

El presente trabajo intentará compartir una experiencia realizada en la comunidad, la cual vislumbrará el fortalecimiento de los vínculos institucionales del Colegio de Psicólogos/as. Dicho trabajo fue llevado a cabo por la Comisión de Género y Diversia del Colegio de Psicólogos de la Provincia de Bs As, distrito XI. La intervención surgió debido a la solicitud realizada por una institución de formación educativa extracurricular de la comunidad al Colegio de Psicólogos/as. El trabajo de la Comisión consistió en dos acercamientos a la institución. Como objetivo puntual nos planteamos en los encuentros la posibilidad de incentivar a que cada une pueda tomar la palabra, expresar su pensamiento sin ser juzgado, ni segregado por ser diferente, rescatando la diversidad, potenciando sus capacidades y adoptando una postura crítica ante la realidad que les atraviesa, apertura que supone el encuentro y la posibilidad de expresar críticamente el pensamiento propio sin censuras.

\section{Palabras clave}

adolescencia | sexualidad y educación | comunicación | malentendido

\section{Cómo citar}

Romero, L., Gentile, J. y Vega, M. (2020).

El malentendido como oportunidad.

Una cuestión de palabras. Revista

de Psicología, 19(1), 179-190. doi:

10.24215/2422572XE049
Recibido

27 ago. 2019

Aceptado

8 mar. 2020

Publicado

17 mar. 2020

\section{Editor}

DOI

$10.24215 / 2422572$ XE049
Nicolás Alessandroni | Facultad de Psicología, Universidad Autónoma de Madrid (España)
ISSN

2422-572X

Licencia

(c) Copyright: Romero, L. et al. Licencia de Cultura Libre CC-BY 4.0

Entidad editora

RevPsi es una publicación de la

Facultad de Psicología (Universidad Nacional de La Plata, Argentina)

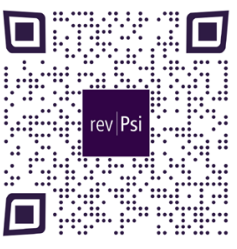

ACCESO ABIERTO DIAMANTE व 


\title{
0 mal-entendido como oportunidade. Uma questão de palavras
}

\section{Resumo}

O presente trabalho procurará compartilhar uma experiência feita na comunidade, que prevê o fortalecimento dos vínculos institucionais do Colégio de Psicólogos. Este trabalho foi realizado pela Comissão de Gênero e Diversidade do Colégio de Psicólogos da Província de Buenos Aires, Distrito XI. A intervenção surgiu devido a um pedido feito por uma instituição de formação educacional extracurricular da comunidade ao Colégio de Psicólogos. O trabalho da Comissão consistiu em duas abordagens à instituição. Como objetivo pontual, durante os encontros propusemos a possibilidade de encorajar cada um a tomar a palavra, a expressar seu pensamento sem ser julgado, nem segregado por ser diferente, resgatando a diversidade, reforçando suas capacidades e adotando uma postura crítica diante da realidade que estão atravessando, uma abertura que implica o encontro e a possibilidade de expressar criticamente seus próprios pensamentos sem censura.

\section{Palavras-chave}

adolescência | sexualidade e educação | comunicação | mal-entendido

\section{The misunderstanding as opportunity. A matter of words}

\begin{abstract}
The present work will try to share an experience made in the community, which will foresee the strengthening of the institutional links of the College of Psychologists. This work was carried out by the Gender and Diversity Commission of the College of Psychologists of the Province of Buenos Aires, District XI. The intervention arose due to a request made by an extracurricular educational training institution of the community to the College of Psychologists. The Commission's work consisted of two approaches to the institution. As a punctual objective, during the meetings we proposed the possibility of encouraging each one to take the floor, to express their thoughts without being judged, nor segregated for being different, rescuing diversity, enhancing their capacities and adopting a critical stance before the reality they are going through, an openness that implies the meeting and the possibility of critically expressing their own thoughts without censorship.
\end{abstract}

\section{Keywords}

adolescence $\mid$ sexuality and education | communication | misunderstanding 


\section{Aspectos destacados del trabajo}

- Se situó el malentendido como posibilidad de la diferencia y no como efecto de la misma.

- Se introdujo el debate como posibilidad de expresión entre los jóvenes y la institución.

- Se favoreció la interrelación entre la comunidad y el Colegio de Psicólogos y Psicólogas del Distrito XI.

- Se destacó la necesidad de la intervención y cuestionamiento institucional acorde a la época.

\section{De la Comisión de Genero y Diversia}

La Comisión de Género y Diversia del Colegio de Psicólogos/as de la Provincia de Buenos Aires, Distrito XI, tiene por objetivo la reunión de colegas que se encuentren interesados en abordar la práctica desde una perspectiva de género. Pensamos a la Comisión como un espacio de debate, de intercambio de conocimientos, este espacio marca un camino a recorrer en la construcción colectiva de saberes y de una práctica por reconocimiento integral sobre el sujeto, donde el género es parte de una perspectiva de derechos humanos.

Son objetivos de la comisión:

- Sensibilizar, visibilizar la perspectiva de género en nuestra práctica profesional.

- Transversalizar la perspectiva de género: capacitacitar e implementar jornadas de reflexión y trabajo sobre diversidad y género para los colegas y para la comunidad.

- Visibilizar los tipos de violencia ya sea física, psicológica, sexual, económica, simbólica; y sus modalidades según en los ámbitos que se manifiesten, violencia doméstica, violencia institucional, violencia obstétrica, violencia mediática, violencia laboral, entre otras (Ley 26.485).

- Promover y difundir las leyes vinculadas a garantizar el pleno respeto de los DDHH de las mujeres y colectivo LGBTTTIQ: Para ello se tiene en cuenta el marco jurídico vigente, partiendo de la Ley de Salud Mental 26.657 y leyes específicas que hacen a la valoración del género, como la Ley Nacional de Protección Integral a las Mujeres 26.485, la Ley 26.618 que reconoce el matrimonio civil igualitario, la Ley 26.743 que establece 
el derecho a la identidad de género de las personas y la Ley de Educación Sexual Integral.

- Generar espacios de contención, de intercambio y de orientación tanto a los/as profesionales como a las Comisiones del Colegio.

- Generar acciones de intervención en lo comunitario sobre violencia de género y con lo que respecta a temáticas de género y diversidad.

- Promover espacios de participación en acciones conjuntas entre integrantes de la Comisión, del Colegio y la comunidad.

- Organizar intervenciones artísticas - urbanas para la visibilización de las temáticas vinculadas a género y diversidad.

\section{Demanda}

La intervención surgió debido a la solicitud realizada por una institución de formación educativa extracurricular de la comunidad al Colegio de Psicólogos/as. Supimos leer la problemática dentro del marco del trabajo que realiza la Comisión de Género y Diversia, orientando el pedido en el marco de la temática. La demanda nos llega de parte de responsables de la institución junto a la madre de un joven asistente a la escuela, transmitiéndonos su preocupación por la situación de malestar ante la "denuncia" pública que había sobre un adolescente varón que asistía a una escuela secundaria por fuera de la comunidad judía. Una compañera de su misma escuela había realizado un escrache por las redes sociales, tildándolo de abusador entre otras cosas. Esta situación repercute de tal manera en las actividades a contraturno que las mujeres se negaron a concurrir a las actividades, talleres e incluso un campamento que estaban organizando si este chico seguía en la institución. En este contexto, las mujeres del grupo etario siguiente al que pertenecia el adolescente en cuestion, exigen a la institucion la desvinculacion del joven denunciado. Es entonces en este momento en que nos llega como Colegio de Psicólogos/as la demanda, el pedido de intervención institucional, ante el desborde de los directivos y muchos de las madres y padres por la situación y no pudiendo encontrar formas eficaces de intervenir.

La mencionada institución, es conocida en la ciudad por ser un movimiento judío, sionista, social y apartidario, que brinda educación no formal y complementaria a los niños/as y adolescentes que pertenecen a la colectividad. Funciona a contraturno de la actividad educativa formal, y tiene como objetivo una educación integral y complementaria para la formación en la vida de los/as adolescentes. Intentan construir un ambiente distinto, de recreación y esparcimiento, en el que se contribuye a formar la idea de grupo y amistad a partir de la puesta en funcionamiento de talleres y actividades deportivas; para trabajar la integración social de todas los jóvenes de la comunidad judía local.

El pedido dejaba ver cierta dificultad de la institución para resolver algunas 
cuestiones tendientes a temáticas de género, sexualidad y comunicación que habían comenzado a circular con intensidad creciente, entre los/as adolescentes que asisten a esa escuela. Dichas dificultades estaban atravezadas por el lema o principio que sostiene a la insticucion, que es la creencia relgiosa, no pudiendo incorporar o hacer lugar a la educacacion sexual integral como contenido necesario dentro de la escuela. Sin embargo a pesar de nocontar con esta formacion especifica y necesaria, pudieron solicitar ayuda a la comisión, para afrontar la problemática.

\section{Marco teórico}

Como todos sabemos, la escuela reúne a diversos grupos de niños/as y a mucho/ as docentes: esta situación hace necesaria la especial atención a las relaciones que entre todos se generan. El desarrollo de vínculos de afecto, de confianza y de respeto requiere plantear espacios específicos para pensar en el otro; requiere "hacer" un lugar para los/as otros/as, para todos/as. Esta es una situación que de algún modo se presenta como un telón de fondo en el escenario de la escuela: todo el tiempo estamos construyendo vínculos; es algo permanente que nos atraviesa a todos y todas, desde el saludo inicial hasta la despedida. Por otro lado, para dedicarse a aprender, se requiere un clima propicio: que todos y todas puedan expresarse, que la palabra circule en el grupo y la escucha sea atenta y respetuosa. Es así como se logran aprendizajes con otros. En esta construcción de un espacio común, con reglas armadas grupalmente, no podemos pasar por alto ni ignorar las situaciones o los conflictos interpersonales o grupales. Es necesario estar permanentemente atentos, con la mirada puesta en lo que sucede; detenerse y abrir espacios de reflexión más o menos sistemáticos para que estas situaciones conflictivas, o que generan malestar, se traten en el aula; hay que "hacerles un lugar" para que podamos avanzar en soluciones de construcción colectiva, por medio del diálogo. Estas cuestiones pueden ser ocasión para trabajar los conflictos de intereses y valores, agresiones físicas, rechazo y discriminación, prejuicios y/o no cuidado en las relaciones con los otros/as, en muchos casos atravesados por cuestiones de género y sexualidad. De otro modo los conflictos permanecen, pueden dañar, aunque los ignoremos, y van en contra de un buen clima de aprendizaje. Un abordaje formativo de los conflictos, reconociendo los derechos y las responsabilidades de cada niño/a y proponiendo formas de resolución y de normas que mejoren la convivencia permitirá fomentar mayores grados de autonomía y desarrollar actitudes orientadas al respeto de los derechos humanos. Por otro lado, el aprendizaje de habilidades para la vida vinculadas al reconocimiento, expresión y valoración de sentimientos puede ser abordado en el aula con consignas simples que contribuyan a que las emociones puedan ser trabajadas en un lugar de encuentro, como la escuela, en el cual se despliegan todo tipo de vínculos e interacciones (Ministerio de Educación, 2009, pp. 77-78).

Que el psicoanálisis tiene algo para decir de los adolescentes lo sabemos desde sus inicios. Fue Freud en Tres Ensayos de Teoría Sexual, en su apartado "Metamorfosis de la pubertad” quien escribió: “(...) con el advenimiento de la pubertad se introducen 
cambios que llevan la vida sexual infantil a su conformación normal definitiva" (Freud, 1905/1993, p. 189).

Como indica Goldber, se trata de "un despertar que alude a lo traumático. Aquello que despierta en este tiempo es una irrupción de lo real, de lo imposible de saber ante la inminencia del encuentro con lo Otro" (2014, p. 89)

En los orígenes mismos del psicoanálisis están los púberes y adolescentes. En palabras de Germán García,

[...] El psicoanálisis nace de la imposibilidad de "superar", de manera tan resuelta y optimista, la tormenta y el empuje de ese periodo crucial de la vida en que la naturaleza y el cuerpo parecen aliarse sin mediación para interpelar las normas que rigen los intercambios sociales. $(2008$, p. 8$)$

Son estos, los/as púberes, los que tienen que arreglárselas con el empuje de lo biológico, pero también vérselas con un empuje discursivo frente al cual responden, precisamente, con la adolescencia. Intentando hacer frente no solo al real traumático ante el encuentro con lo Otro, sino también con lo que el Otro social tiene para decir sobre ello.

Sabemos que hay un insalvable malentendido desde el origen mismo del sujeto. Un malentendido propio de la división subjetiva que, si bien da lugar al gran problema inherente al ser hablante, es también condición de posibilidad para que cada sujeto encuentre su forma de responder al mismo. Sibien consideramos que cada adolescente buscará responder a sus interrogantes y orientarse en relación a la construcción de su propio arreglo con el goce, no podemos desconocer que existe un orden social, un ideal social que marca un supuesto destino posible para los adolescentes en cada época y contexto. Un discurso social que cambia, que no es estático y que en esta época que atravesamos tiene ciertas particularidades que pueden favorecer, pero también desorientar aún más a estos jóvenes.

¿Cuáles son entonces las características de la época? Asistimos a un tiempo donde la relación con el otro se ve continuamente cortocircuitada. Los ideales y modelos que han regido hasta hoy como el modelo patriarcal, se encuentra en una clara decadencia, dando lugar a la gestación de nuevos interrogantes y modelos. La lucha de distintos sectores por la despenalización del aborto y la incidencia del crecimiento de los feminismos y disidencias genera la necesidad de inventar e incluirles en ese invento.

Los jóvenes adolescentes tienen en claro estos movimientos y son activistas del cambio. Lo gestionan, lo sostienen. Se muestran decididos a llevar a cabo estas transformaciones.

Celebramos dicho cambio y su consecuente efecto de destitución del modelo heteronormativo que hemos sostenido desde que tenemos uso de razón, pero somos conscientes tambien de que es necesario construir modelos que sostengan y 
favorezcan la singularidad.

Lejos de criticar los cambios sociales y dedicarnos a analizarlos, creemos que, desde la psicología y desde el psicoanálisis, tenemos que estar a la altura de estos avatares y transformaciones. Como nos dice Laurent con su conceptualización del "analista ciudadano":

[...] El analista, más que un lugar vacío, es aquel que ayuda a la civilización a respetar la articulación entre normas y particularidades individuales. [...] Así, con otros, ha de ayudar a impedir que en nombre de la universalidad o de cualquier universal, ya sea humanista, o anti humanista, se olvide la particularidad de cada uno. [...] No se trata de limitarse a cultivar, a recordar la particularidad, sino de transformarla en algo útil. (Laurent, 2000, pp. 117-118)

Es desde este esta perspectiva, teniendo como marco las leyes que nos regulan y nos permiten dar lugar a estos cambios, que intervenimos en esta institución que en muchos momentos el sujeto adolescente se refirió a un "individuo" particular, como en otras, al grupo de adolescentes como sujetos en relación con la institución.

Si bien el pedido fue explícitamente realizado por una situación puntual ocurrida entre los grupos etarios de mayor edad de la institución y con cierto temor por lo que este conflicto pudiese generar a un nivel institucional mayor. Supimos leer que lo que sucedía iba mucho más allá del pedido. Pudiendo dar cuenta del malentendido propio existente entre aquello que se pide, se demanda y la intervención propicia. Entendimos que lo que estaba en juego era la dificultad institucional para dar cuenta del impacto de lo social, sexual y generacional que se hacía presente en dichos adolescentes, pudiendo cuestionar las herramientas con la que la institución misma contaba.

Desde la Comisión pudo recortarse la problemática haciendo hincapié en los ejes mencionados anteriormente, proponiendo así un trabajo en conjunto con la institución, a partir de la utilización de técnicas grupales, aportes de la Psicología Comunitaria y el Psicoanálisis, además de servirnos de la Ley 26.150 de Educación Sexual Integral, en sus artículos 8b, 9b y 9c.

En este sentido, entendemos que la ESI es la puerta de entrada de la Educación Sexual Integral a la escuela secundaria porque presenta la posibilidad de construir diversas estrategias que pueden implementarse para iniciar, fortalecer y/o dar continuidad al desarrollo de la Educación Sexual Integral en la institución escolar.

Muchas veces algunos posicionamientos desde las instituciones adquieren un rango de certeza que resulta ser luego una imposibilidad trabajar estos temas. Pero en estas ocasiones, por debajo de estas se ocultan temores basados en creencias, estereotipos, tabúes, prejuicios y modelos sobre la sexualidad y la educación sexual que fuimos naturalizando a lo largo de nuestra historia personal. De tal modo, que la intervención institucional en esta temática, debe necesariamente recoger esta información desde una escucha activa y propiciando un dispositivo de trabajo que logre la puesta en común de la palabra, y posibilite la visibilización de los saberes 
sobre la sexualidad que habitan y atraviesan esa institución -docentes y estudiantes-, tal como lo sugiere la propia Ley.

\section{Técnica utilizada: taller}

La metodología para la intervención consistió en la realización de dos talleres, que buscaron ofrecer un espacio de trabajo grupal en el cual se crean o modifican comportamientos y/o pensamientos. Consideramos que conforman una metodología adecuada ya que brindan un espacio de producción y reflexión, donde los estudiantes son capaces de plasmar y proyectar sus diversos puntos de vista en producciones tanto creativas como orales y escritas.

Si bien el primer acercamiento de la institución educativa al Colegio de Psicólogos/ as refirió a un hecho en particular, la escucha de la Comisión apuntó a ir más allá de esta dándole la voz y participación a los adolescentes que concurren allí, pensando en ellos como sujetos de derechos desde una perspectiva integral.

El trabajo de la Comisión consistió en dos acercamientos a la institución, previa reunión con el equipo directivo, los cuales permitieron contextualizar la situación de la institución y la de los adolescentes.

\section{Primer taller}

La posición de la Comisión en este taller apuntó a ir más allá de la demanda dándole la voz y participación a los adolescentes que concurren allí, pensando en ellos como sujetos de derechos desde una perspectiva integral.

Desarrollo. La dinámica del taller consistió en trabajar con los jóvenes según los grupos de edad con los que ellos ya vienen trabajando 13 y 14 años; $15 ; 16$ y por otro lado los coordinadores que tienen más de 17 años, estos últimos son estudiantes egresados de dicha institución que en su último año se forman en liderazgo para coordinar los espacios de formación.

- Comenzamos el primer encuentro bajo la pregunta ¿por qué creen que estamos acá?

- Está pregunta abierta permitió que visibilizar los imaginarios que circulaban en torno a nuestra presencia y ver si a su vez podía ponerse en juego la demanda de la institución. Según cada grupo fueron surgiendo diferentes respuestas por un lado que íbamos a dar un taller sobre género y en el grupo de los coordinadores tenían más en claro nuestra presencia allí.

- Luego se llevó adelante la actividad que consistía en la lectura de una viñeta sobre la relación de dos amigas de la infancia de 14 años, en la cual una de ellas tenía dudas sobre su identidad sexual y sus elecciones. Ella 
comienza a sentirse atraída por su amiga y un día en su casa la acaricia. Frente a la falta de rechazo de su amiga inicia una escena sexual. Esta se enoja, le quita las manos de encima y al otro día en la escuela lo comenta. Empiezan a apartarla de los espacios, motivo por el cual interfieren las autoridades de la escuela.

- Luego de la lectura se dieron preguntas orientadoras para trabajar donde se los invitaba a tomar las diferentes posiciones de los actores intervinientes en la viñeta.

- Al final se dio un espacio de plenario con todos los grupos donde se compartió lo trabajado. Allí fue interesante ver cómo según cada edad iban teniendo diferentes lecturas de la escena, algunos veían la situación de abuso, otros resaltaban el lugar de lo íntimo y hasta donde el "escrache" tiene lugar, nuestra intervención fue darle lugar a que siempre es importante resaltar el lugar de las instituciones como recurso. También surgieron inquietudes acerca de la identidad sexual.

El momento del plenario resulto interesante. El grupo que estaba en desacuerdo con la presencia del joven en la institución, pudo exponer su pensamiento a pesar de haber sido quien proponían el escrache como medio, sosteniendo una posición de resistencia frente a nuestra presencia.

\section{Segundo taller}

Previo a este taller tuvimos una reunión nuevamente con la directora y dos madres de la institución. Aquí el pedido era diferente ya que la demanda surgió debido a que un grupo de chicas de la institución estaba dejando de lado a otra chica por tener una relación con un chico que había, en palabras de las jóvenes, "acosado a una compañera". Quien tenía la relación había hablado con el joven y le había creído su versión ya que además había pedido perdón a la joven involucrada en la escena.

Se conversó sobre las repercusiones del encuentro anterior y cuáles habían sido los efectos en los adolescentes. Los coordinadores comentaron que los jóvenes no habían podido apreciar el objetivo del taller ya que no tenía que ver con lo que ellos demandaban. En cambio los coordinadores resaltaron la importancia de estos espacios donde poder encontrar herramientas para abordar las problemáticas que se le presentaban en lo cotidiano. De este encuentro emergieron algunos ejes de trabajo entre los que se destacaron: el respeto, las posiciones diferentes, la vincularidad, el cuidado/autocuidado.

Teniendo como premisas estas preguntas:

- ¿Cuál es el proceso por el cual se reconoce el error del otro?

- ¿No alcanza con hacerse responsable del acto realizado? 


\section{- ¿Dónde está el límite?}

Pensamos un taller en el cual abordar los modos de comunicarse con el otro, trabajar sobre la diversidad y el respeto por pensar, sentir y hacer en la diferencia. Las consignas del taller apuntaron a pensar(se) con el otro. Nos planteamos la posibilidad de que el trabajo grupal producido en el ámbito del taller pudiera tener implicancias a nivel personal; es decir que el trabajo grupal pudiese de alguna forma conmover o transformar en algún sentido los pensamientos individuales de cada sujeto integrante del colectivo.

Este objetivo, lejos de buscar producir un fenómeno de masa entrelos/as participantes, intentaba partir de la reflexión propia y el compartir con otros/as teniendo como punto de llegada la transformación y creación propia del pensamiento dialectico, en el transcurso del taller.

El disparador de este fue la pregunta: ¿Cuándo emitimos una opinión en que nos basamos? Luego se mostraron unas imágenes que podían tener varios significados y entre ellos ir construyendo el sentido. En un tercer momento se pasó a un espacio de psicodrama donde los adolescentes representaban alguna escena y otros representaban tipos de escucha.

- El generador de réplica (escucha lo suficiente para generar su réplica)

- El aconsejador (salta rápido para dar consejos no solicitados)

- El interruptor (ansioso por decir sus palabras)

- El escuchador lógico (obvia sentimientos)

- El que se cuelga del relato ("lo que me pasó a mí...")

- El falso escuchador (el que finge escuchar)

Luego cada joven comentó cómo se había sentido en ese rol, manifestando la incomodidad de hacer algo con lo cual no se sentían identificados. En el momento del plenario se compartió lo trabajado en el cual quienes habían tenido la escena del malentendido pudieron expresarse.

\section{Algunas reflexiones}

Se tomó como disparador el trabajo sobre la comunicación partiendo de la premisa de que toda comunicación con el otro supone un malentendido, pero considerando que hay ciertos tipos de comunicaciones que acentúan aún más la imposibilidad de relacionarse entre las personas, en este caso los jóvenes.

Solemos considerar que el malentendido puede ser producto de una mala manera o forma dificultosa de expresar aquello que queremos decir. Las teorías de la comunicación incluso han hecho hincapié en las distintas formas en las cuales la 
comunicación sería un hecho posible. Es decir que podríamos expresar aquello que queremos decir y que el otro lo entienda en el sentido mismo en que fue emitido:

[...] Mediante el instrumento del lenguaje se instaura cierto número de relaciones estables, en las que puede ciertamente inscribirse algo mucho más amplio, algo que va mucho más lejos que las enunciaciones efectivas (Lacan, 1969/2009, pp. 10-11).

El psicoanálisis habla de discursos. Ha podido realizar contribuciones en relación con esta cuestión a partir de la elaboración y el análisis de los 4 discursos (discursos del amo, histérico, universitario y analítico), dando cuenta que los mismos suponen lugares fijos que van siendo ocupados por distintos elementos que, según como se relacionen entre sí, establecen posiciones subjetivas y hechos de discurso diferentes.

El sujeto surge de un malentendido y por ser sujeto hablante ingresa al mundo del lenguaje de esta forma. Al hablar produce hechos de discurso, y al no hacerlo, también. Si tomamos estos hechos discursivos como intentos de comunicación entre los sujetos, podemos ver que el lenguaje tiene un límite. Un límite propio del significante como tal, que no puede decirlo todo, que mata a la cosa al ponerle un nombre dejando un resto imposible de recuperar.

En este sentido es que podemos decir que, al emitir un mensaje, quien lo recibe no puede más que intentar interpretarlo. Evidenciando que la comunicación entre los diferentes actores sea de alguna forma impura o se vea alterada por la subjetividad de cada uno/a. Distancia propia entre el decir y lo dicho que establece entre ambos una hiancia imposible de erradicar.

A pesar de considerar entonces, que la comunicación per se no es posible, quizás es necesario poder dar cuenta que podemos sostener espacios discursivos y transmitir ideas y pensamientos, en la medida en que estemos dispuestos a soportar dicho malentendido o dicha falla inherente al ser hablante. ¿De qué forma? Respetando al otro e intentando dar lugar a la singularidad; es decir, poder escuchar sin intentar cambiar o modificar lo que el otro/a piensa. Si bien el sujeto surge de un malentendido y por ser sujeto hablante ingresa al mundo del lenguaje de esta forma, es desde esta perspectiva que quisimos poner a jugar el malentendido a partir de las técnicas utilizadas.

Dichas intervenciones apuntaron a trabajar desde lo grupal, tomando aportes del campo del Psicoanálisis y la Psicología Comunitaria pensando al grupo como un proceso abierto, un devenir y un lugar privilegiado. Se organizaron grupos de trabajo según las edades de los mismos, apuntando a desplegar en cada uno la creatividad, la escucha y el respeto, así como hacer lugar a los interrogantes e inquietudes que la situación planteada generase.

Como objetivo puntual nos planteamos en los encuentros la posibilidad de incentivar a que cada uno pueda tomar la palabra, expresar su pensamiento sin ser juzgado, ni segregado por ser diferente, rescatando la diversidad, potenciando sus capacidades 
y adoptando una postura crítica ante la realidad que los atraviesa, apertura que supone el encuentro y la posibilidad de expresar críticamente el pensamiento propio sin censuras.

Concluimos el encuentro en un espacio de plenario que permitió evidenciar lo trabajado en los talleres y compartirlo con el colectivo. Sosteniendo la espera y respetando la diferencia. Rescatando entonces que lejos de llegar a un consenso y respuesta unívoca, lo que se pudo escucharse fueron diversas inquietudes y preguntas que apuntaban a la necesidad de seguir generando este tipo de espacios, ricos para ellos en términos de expresión y disenso y, para la institución en términos de la apertura y la necesidad de interrogarse y renovarse activamente.

De lo trabajado surgió explícitamente la necesidad de incluir como parte de la institución, un espacio de reflexión y de formación en lo respectivo a la ESI , educación sexual integral. Considerado como fundamental para dar espacio a las manifestaciones que los avatares del crecimiento y el compartir grupal entre niños/ as y adolescentes expresan.

Será todo un desafío para la institución pensar el como incluir este contenido dentro de su formación, contando por supuesto con el apoyo de la Comisión en todo momento.

\section{Referencias}

Freud, S. (1905/1993). Tres ensayos de teoría sexual. En J. L. Etcheverry (Trad.), Obras completas. Tomo VII (pp. 109-224). Amorrortu.

García, G. (2008). El embrujo de la pubertad. En M. Recalde (Ed.), Púberes y adolescentes (pp. 7-12). Grama.

Goldber, S. (2014). Pubertad. Camino a la sexuación. En VV.AA., Psicoanálisis con niños y adolescentes $N^{\circ} 4$ (pp. 89-92). Grama.
Lacan, J. (1969/2009). El Seminario. Libro 17. El reverso del psicoanalisis. Paidós.

Laurent, E. (2000). Psicoanálisis y salud mental. Tres Haches.

Ministerio de Educación de la República Argentina. (2009). ESI para la educación primaria. Contenido y propuestas para el aula. Presidencia de la Nación Argentina. 